Summer Meeting, 4-6 July 2011, 70th Anniversary: From plough through practice to policy

\title{
Systematic review comparing the effectiveness of self help $v$. group weight management programme in weight loss
}

\author{
N. Khasteganan and A. Tsiami \\ Faculty of Health and Human Science, TVU, Boston Manor Road, Brentford TE8 9GA, UK
}

\begin{abstract}
The research is a systematically review of literature comparing the effectiveness of group weight loss programme (GWLP) and self-help weight loss programmes (SHWLP). Research method was systematically searching databases Cochrane library, PUBMED and MEDLINE from 2000 until June 2010. Selection criteria were randomised controlled trials include either GWL or SHWLP or both. Obese or overweight adults aged between 18 and 65, BMI between 25 and 40. For data collection and analysis, the reviewer used standardised tools to extract data and rate every study's methodological quality. Thirteen articles were included, six were in the SHWLP and nine were in GWLP. The mean methodological quality score was 19.5 in both groups (the minimum score was 6 , while the maximum score was 26 , see Table 1). The mean weight loss (kg) for SHWLP was 1.19, while GWLP had 7.08. The GWL intervention had a mean follow up months of 11.6, while the SHWLP had 13.5 (see Table 2). Higher weight loss was noticed in the GWLP as compared with the SHWLP (see graph 1). The final results should be used with care because of lack of Meta analysis in the review as the data were inhomogeneous.
\end{abstract}

Table 1. Randomised controlled trials reporting the self and group weight management programme in weight loss.

\begin{tabular}{|c|c|c|c|c|c|c|c|c|c|c|c|}
\hline SHWLP & $\begin{array}{c}\text { Completers } \\
(\%)\end{array}$ & Studies'score & $\begin{array}{l}\text { Duration } \\
\text { (m) }\end{array}$ & Size & $\begin{array}{l}\text { Weight } \\
\text { loss (kg) }\end{array}$ & GWLP & $\begin{array}{c}\text { Completers } \\
(\%)\end{array}$ & Studies' score & $\begin{array}{l}\text { Duration } \\
\quad(\mathrm{m})\end{array}$ & Size & $\begin{array}{l}\text { Weight } \\
\text { loss (kg) }\end{array}$ \\
\hline Welsh et al. ${ }^{(1)}$ & 31 & $15 * * *$ Moderate & 6 & n 29 & $1.7 \pm 2$ & Corbalán et al. ${ }^{(2)}$ & 89 & $18 * * * *$ Strong & 8 & n 1406 & $7.8 \pm 5.58$ \\
\hline Helsel et al. ${ }^{(3)}$ & 62 & $15 * * *$ Moderate & 4 & $n 21$ & $3.9 \pm 5.3$ & Truby et al. ${ }^{(4)}$ & 89 & $16^{* * *}$ Moderate & 2 & $n 58$ & $4.7 \pm 3.2$ \\
\hline Byrne et al. ${ }^{(5)}$ & 82 & $19 * * * *$ Strong & 8 & $n 33$ & $2.6 \pm 3.6$ & Teixeira et al. ${ }^{(6)}$ & 70 & $15 * * *$ Moderate & 4 & $n 158$ & 5.1 \\
\hline Womble et al. (7) & 66 & $20 * * * *$ Strong & 13 & n 24 & $3.3 \pm 4.1$ & Latner et al. ${ }^{(8)}$ & 23 & $16 * * *$ Moderate & 60 & $n 128$ & $15.6 \pm 1.5$ \\
\hline Heshka et al. ${ }^{(9)}$ & 75 & $24 * * * * *$ Strong & 24 & $n 212$ & $0.2 \pm 6.5$ & Harvey et al. ${ }^{(10)}$ & 95 & $16 * * *$ Moderate & 3 & $n 46$ & $6.4 \pm 5.6$ \\
\hline \multirow[t]{4}{*}{ Heshka et al. } & 81 & $24 * * * * *$ Strong & 6 & n 212 & $1.4 \pm 4.7$ & Heshka et al. ${ }^{(9)}$ & 70 & $24 * * * * *$ Strong & 24 & $n 211$ & $2.9 \pm 6.5$ \\
\hline & & & & & & Heshka et al. (11) & 82 & $24 * * * * *$ Strong & 6 & $n 211$ & $4.8 \pm 5.6$ \\
\hline & & & & & & Truby et al. (12) & 81 & $21 * * * * *$ Strong & 6 & $n 58$ & $6.6 \pm 5.6$ \\
\hline & & & & & & Waleekhachonloet et al. ${ }^{(13)}$ & 89 & $26 * * * * *$ Strong & 12 & $n 65$ & $3.7 \pm 0.58$ \\
\hline
\end{tabular}

Table 2. Comparison between mean weight loss and duration of follow up among self-helping and weight management group

\begin{tabular}{lcccc}
\hline Group & $\begin{array}{c}\text { Total } \\
\text { participants }\end{array}$ & $\begin{array}{c}\text { Mean articles } \\
\text { score (min-max) }\end{array}$ & $\begin{array}{c}\text { Mean follow } \\
\text { up (months) } \\
\text { (min-max) }\end{array}$ & $\begin{array}{c}\text { Mean weight } \\
\text { loss (kg) } \\
(\text { min-max) }\end{array}$ \\
\hline Self-helping & 531 & $19.5(15-24)$ & $13.5(4-24)$ & $1.19(0.2-3.9)$ \\
Group-managing & 2341 & $19.5(15-26)$ & $11.6(2-60)$ & $7.08(2.9-15.6)$ \\
\hline
\end{tabular}

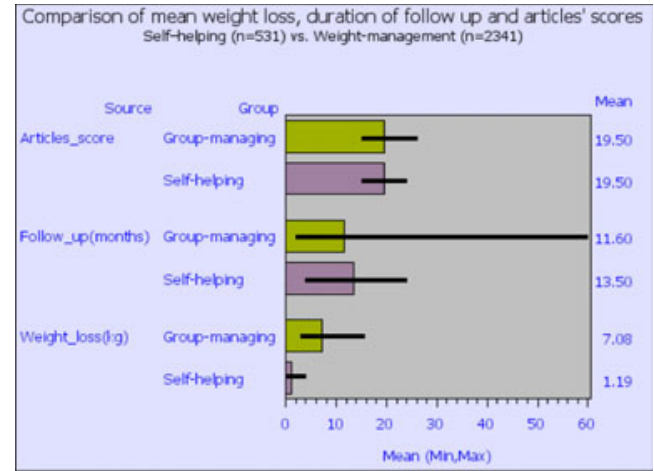

Graph 1. Systematic review results' graph

1. Welsh EM, Sherwood NE, VanWormer JJ et al. (2009) Is frequent self-weighing associated with poorer body satisfaction? Findings from a phonebased weight loss trial. $J$ nutr educ behav 41 (6), 425-428.

2. Corbalán MD, Morales EM, Canteras M et al. (2009) Effectiveness of cognitive-behavioral therapy based on the Mediterranean diet for the treatment of obesity. Nutrition $25(7-8), 861-869$.

3. Helsel DL, Jakicic JM \& Otto AD (2007) Comparison of techniques for self-monitoring eating and exercise behaviors on weight loss in a correspondence-based intervention. J Am Diet Assoc 107 (10), 1807-1810.

4. Truby H, Hiscutt R, Herriot AM et al. (2008) Commercial weight loss diets meet nutrient requirements in free living adults over 8 weeks: A randomised controlled weight loss trial. Nutr J 7, No. 25.

5. Byrne NM, Meerkin JD, Laukkanen R et al. (2006) Weight loss strategies for obese adults: Personalized weight management program vs. standard care. Obes J 14 (10), 1777-1788.

6. Teixeira PJ, Going SB, Houtkooper LB et al. (2004) Pretreatment predictors of attrition and successful weight management in women. Int J Obes Relat Metab Disord 28 (9), 1124-1133.

7. Womble LG, Wadden TA, McGuckin BG et al. (2004) A Randomized Controlled Trial of a Commercial Internet Weight Loss Program. Obes Res $J$ 12, 1011-1018.

8. Latner JD, Wilson GT, Stunkard B, AJ et al. (2002) Self-help and long-term behavior therapy for obesity. Behav Res Ther 40 (7), 805-812.

9. Heshka S, Anderson JW, Atkinson RL et al. (2003) Weight loss with self helps compared with a structured commercial program, pub J Am Med Assoc 289 (14), 1792-1798.

10. Harvey-Berino J, Pintauro S, Buzzell P et al. (2002) Does using the Internet facilitate the maintenance of weight loss? Int J Obes 26, $1254-1260$.

11. Heshka S, Anderson JW, Atkinson RL et al. (2000) Weight loss with self-help compared with a Structured Commercial Program a Randomized Trial. Am J Med 109 (4), 282-287.

12. Truby H, Baic S, deLooy A et al. (2006) Randomised controlled trial of four commercial weight loss programmes in the UK: initial findings from the BBC diet Cite this article as: BMJ, doi:10.1136/bmj.38833.411204.80. Available at http://www.ajcn.org. (accessed 17 February, 2010).

13. Waleekhachonloet OA, Limwattananon CH, Limwattananon S et al. (2007) Group behavior therapy versus individual behavior therapy for healthy dieting and weight control management in overweight and obese women living in rural community. Obes Res Clin Pract 1 (4), $223-232$. 